\title{
Adherence to Guidelines During Follow-up of Endometrial Cancer: Analysis of French Health Insurance Database
}

\author{
JULIETTE PHELIPPEAU ${ }^{1,2}$, ROMAN ROUZIER $^{2,3}$ and MARTIN KOSKAS ${ }^{1,2}$ \\ ${ }^{1}$ Gynecologic Oncology, Bichat University Hospital, Paris Diderot University, Paris, France; \\ ${ }^{2}$ EA 7285, Versailles Saint Quentin University, Montigny Le Bretonneux, France; \\ ${ }^{3}$ Department of Surgical Oncology, René Huguenin Centre, Saint-Cloud, France
}

\begin{abstract}
Background/Aim: Recommendations for endometrial cancer (EC) follow-up after treatment include regular clinical examinations without further systematic investigation. The objectives of our study were to examine adherence to follow-up guidelines in patients who underwent surgery for EC and identify associated variables. Patients and Methods: Patients who underwent surgery for EC between 2005 and 2014 were identified among the Echantillon Généraliste des Bénéficiaires. The adherence to guidelines was defined by fewer than two investigations performed, including vaginal smear, CA 125 evaluation and imaging during two postoperative years. Results: Among the 314 women identified, a gap between recommendations and practices was observed in $48.1 \%$. This gap was more frequently observed in patients who underwent surgery before 2011, in private institutions and in institutions performing less than 20 hysterectomies for EC per year and who had Long Duration Disease declaration for EC. Conclusion: A gap between recommendations and practices for the follow-up was observed in almost half of the patients with EC. Updating the recommendations in 2010 may have improved compliance with the recommendations.
\end{abstract}

Endometrial cancer (EC) is the fourth most common cancer in women in Europe, with an estimated incidence of 88000 cases in 2012 (1). Because early symptoms lead to consultation, $70 \%$ of EC cases are diagnosed at early stages (International Federation of Gynecology and Obstetrics (FIGO) stage I or II). In such cases, the 5-year survival is $90 \%$. Recurrence occurs in 10 to $15 \%$ of women treated for EC, especially in the first three years, which justifies close early follow-up (2). Overall, approximately $75 \%$ of recurrences are symptomatic (3). The

Correspondence to: Dr. Martin Koskas, Department of Obstetrics and Gynecology, CHU Bichat Claude Bernard, 46, rue HenriHuchard, 75018 Paris, France. Tel: +33 0140258080, Fax: +33 0140256757,e-mail: martin.koskas@wanadoo.fr

Key Words: Endometrial cancer, follow-up, guidelines. conventional reasons for follow-up of treated cancer patients include providing reassurance and early diagnosis of recurrence (4). Currently, European recommendations from the European Society for Medical Oncology (ESMO) for EC follow-up consist of a clinical examination for signs of recurrence. If there is no symptom of recurrence, it is not recommended to perform further investigations (2). The latest French professional recommendations, concordant with ESMO recommendations, were published in November 2010. The previous follow-up recommendations for EC were similar and were published in 2000 (5).

Few studies have addressed follow-up after the treatment of EC. Two systematic reviews $(6,7)$ documented evidence for the utility of follow-up investigations in EC. Both concluded that follow-up should be practical and directed by symptoms and the results of pelvic examinations. Given the low risk of recurrence, vaginal cytology can be omitted, resulting in reduced healthcare costs (8). It appears that visual inspection is sufficient since positive cytology is merely diagnosed in cases of symptomatic recurrence (9-11).

In medical practice and especially in oncology, recommendations are necessary due to cost issues, the availability of resources and the individual impact (psychological) on patients. Few studies have evaluated the adherence to recommendations in actual clinical practice, especially for the follow-up. Follow-up practices have never been reported for EC. A Dutch study reported a care overconsumption in $27 \%$ of cases after cancer (12).

The main objective of our study was to describe the gap between recommendations and practices in the follow-up of patients who underwent surgery for EC in France. The second objective was to identify explanatory factors for nonadherence to recommendations.

\section{Patients and Methods}

Study population. We performed our study with the Echantillon Généraliste de Bénéficiaires (EGB), a national database that records the consumption of care. The EGB is a permanent representative sample of about 1 percent of the national population covered by 
French health insurance constituted by the Health insurance national company (CNAM) and established in 2003 (13).

In the EGB, databases available from January 2005 to December 2014 were used to identify cases and characteristics of hospital stays. We also extracted socio-demographic characteristics of patients, such as age, care department, health insurance regime, date of death and other diseases completely covered by the national insurance.

\section{Inclusion criteria}

Inclusion criteria were:

- Women who have had surgical treatment: total hysterectomy with bilateral salpingo-oophorectomy after a diagnosis of EC between January 2005 and December 2014.

The reference cancer date was the surgery date for EC.

Exclusion criteria were:

- Patients with another cancer diagnosed before or after EC.

- Patients with probable EC recurrence. Those patients were excluded if they underwent a pelvectomy and/or chemotherapy and/or radio brachytherapy for EC more than 9 months after surgery.

Indeed, these patients received imaging tests for diagnostic purposes that do not constitute a gap between recommendations and practices.

Indicators for adherence to guidelines. According to the European and French recommendations, systematic investigations are not indicated for the follow-up of patients who underwent surgery for EC unless a recurrence is suspected. The gap between recommendations and practices for the follow-up of patients who underwent surgery for EC can therefore be defined by unjustified additional investigations. The following postoperative investigations were investigated:

- Vaginal smear

- CA 125 levels

- Imaging tests: thoracic, abdominal and pelvic CT scan, thorax or abdominal radiography and abdominal ultrasound.

The primary endpoint was the adherence to recommendations during the first 2 years after surgery for EC. Non-adherence to recommendations was defined when the patient had at least two exams among vaginal smear, CA 125 and imaging test during the two postoperative years. In order to exclude imaging related to the exploration of postoperative complications, we considered complementary investigations from three months to two years after surgery only. Moreover, we compared overall survival according to adherence to recommendations in order to evaluate the comparability of the two groups.

Statistical analysis. The practices of EC follow-up through the three indicators were examined.

The log rank test was used to compare overall survival according to adherence to recommendations. The second part of the study used a random intercept multilevel regression to determine explanatory factors to estimate the contextual effect in an individual analysis. The two levels were individual and institutional levels. Variables with a significance level of less than 0.20 in the univariate analysis were included in the multivariate analysis.

The statistical software used for the study was SAS enterprise guide for the extraction of data from the interface of the EGB and $\mathrm{R}$ for statistical analysis (http://lib.stat.cmu.edu/R/CRAN).
Table I. Epidemiological and clinical characteristics of the 314 women included.

\begin{tabular}{lc}
\hline $\mathrm{N}=314$ & Mean $[95 \% \mathrm{CI}]-\mathrm{n}(\%)$ \\
\hline Age [years, 95\%CI] & $66.3[65.1-67.5]$ \\
Mean follow up [months, 95\%CI] & $55.8[53.7-57.9]$ \\
Type of institution & \\
$\quad$ Public & $155(49.4)$ \\
Private & $152(48.4)$ \\
NA & $7(2.2)$ \\
Universal medical health care & $26(8.3)$ \\
Hypertension & $26(8.3)$ \\
Diabetes & $55(17.6)$ \\
Hysterectomy route & \\
Laparotomy & $258(82.2)$ \\
Laparoscopy & $56(17.8)$ \\
Lymph nodes resection associated & $174(55.4)$ \\
Adjuvant treatment & $110(35)$ \\
\hline
\end{tabular}

Data are expressed as n (\%) unless otherwise specified. CI: Confidence interval.

\section{Results}

Characteristics of the population. A total of 547 cases of EC were identified in the EGB between 2005 and 2014. Of these 547 cases, 405 patients had at least a total hysterectomy; 116 women $(21 \%)$ did not have surgical treatment, and $26(4.8 \%)$ had another diagnosis associated with hysterectomy for EC (mainly ovarian cancer). Among the 405 patients, 18 patients were excluded because of the probability of recurrence, and 73 were excluded because they had associated cancers.

A total of 314 women who underwent a total hysterectomy between January 2005 and December 2014 were finally included in the study (Figure 1).

Patient characteristics are shown in Table I. The mean follow-up period in the study was 55.8 months [95\% CI $=53.7-57.9]$. The mean age of patients was 66 years old $[95 \% \mathrm{CI}=65-68]$. Half of the patients were treated in public institutions. Approximately $18 \%$ of patients had associated diabetes. A total of $17.8 \%$ of patients underwent laparoscopic surgery, and 35\% received adjuvant therapy (radiation and/or chemotherapy). The overall survival at 2 years and 5 years was $87 \%$ and $78 \%$, respectively (Figure 2).

Adherence to the guidelines. Among the 314 cases, 60 $(19.1 \%)$ had at least one postoperative vaginal smear with a total of 135 vaginal smears performed within the 2-year follow-up. Overall, 122 patients (38.9\%) had at least one CA 125 assessment post operatively with a total of $582 \mathrm{CA} 125$ assessments within the 2-year follow-up. A total of 196 patients $(62.2 \%)$ underwent postoperative imaging at least once. Imaging was performed 935 times within the 2-year follow-up. Overall, 163 patients $(51.9 \%)$ had no or one test among the three markers during the two years of 


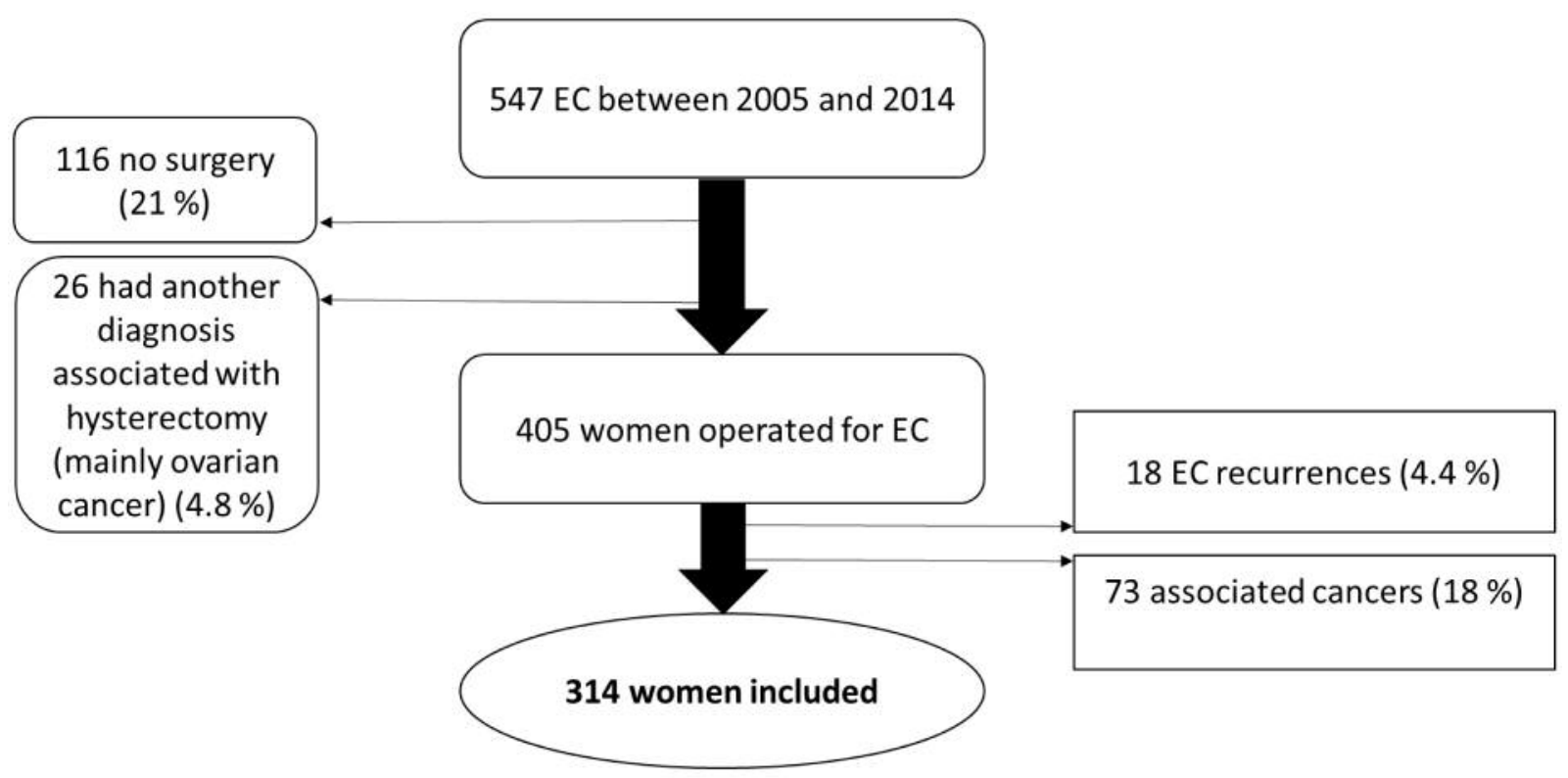

Figure 1. Flow chart of the study.

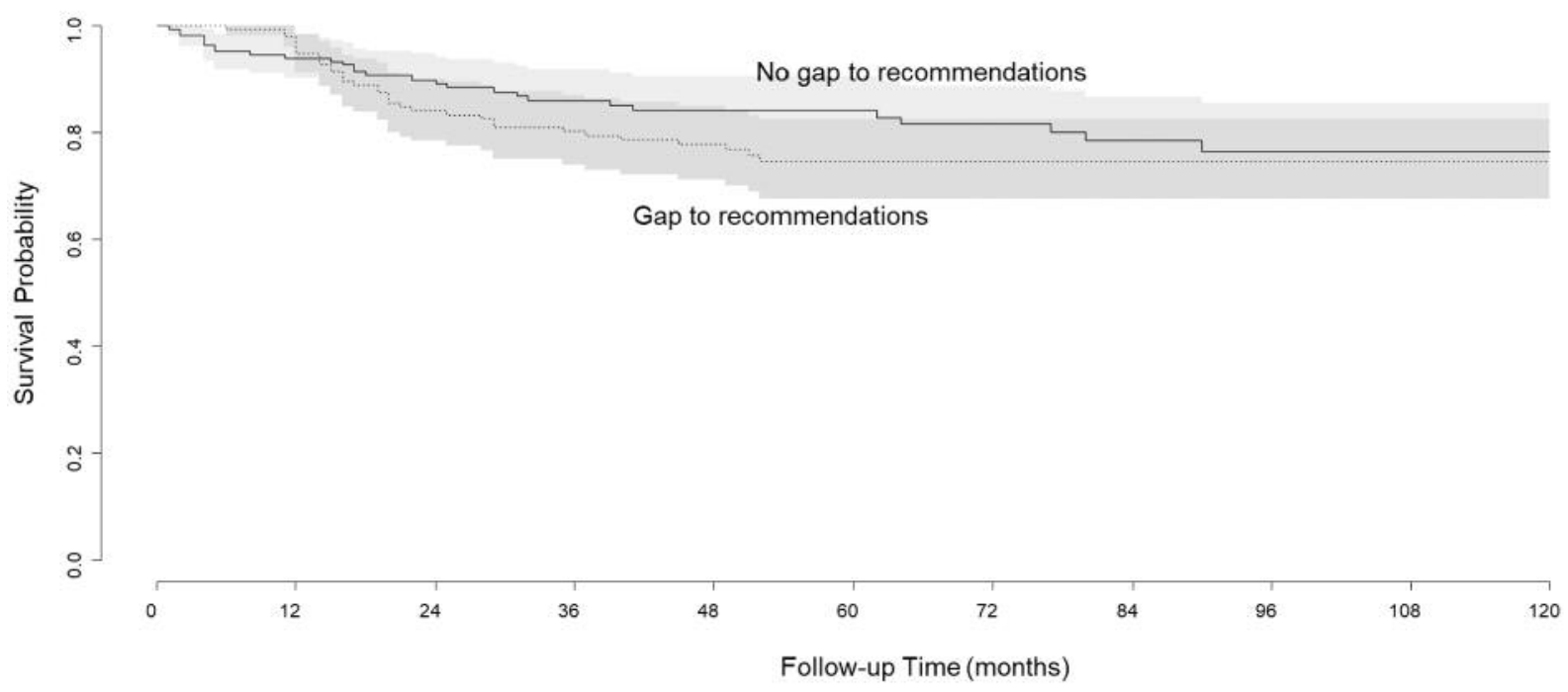

Figure 2. Kaplan-Meier curve: Overall survival of the women with and without a difference to recommended practices in follow-up after EC treatment $(p=0.31)$.

postoperative care, and their follow-up was considered to be in accordance with the guidelines. A total of 151 patients $(48.1 \%)$ had at least two vaginal smears and/or CA 125 evaluations and/or imaging within the two years of followup and were considered to have a gap between the recommendations and practices.

Comparison of overall survival according to the adherence with guidelines. Figure 2 shows that there was no difference in overall survival between the two groups; overall survival after 3 years was $80 \%$ for the 151 women with a gap between recommendations and practices, and the overall survival after 3 years was $84 \%$ for the remaining 163 patients $(p=0.31)$.

Analysis of determinant factors. The results of the univariate and multivariate multilevel analyses are presented in Table II. The declaration of LDD for EC significantly increased the risk of a gap between recommendations and practices during 
Table II. Determinant factors of difference in practices (Univariate and multivariate multilevel analysis).

\begin{tabular}{|c|c|c|c|c|}
\hline & \multicolumn{2}{|c|}{ Univariate } & \multicolumn{2}{|c|}{ Multivariate } \\
\hline & OR $[95 \% \mathrm{CI}]$ & $p$-Value & OR $[95 \% \mathrm{CI}]$ & $p$-Value \\
\hline \multicolumn{5}{|l|}{ Individual factors } \\
\hline Age $>65$ years old & $0.98[0.87-1.09]$ & 0.72 & - & - \\
\hline Universal health care insurance & $0.86[0.75-0.97]$ & 0.14 & $0.88[0.68-1.09]$ & 0.23 \\
\hline Long duration disease declaration & $1.21[1.10-1.32]$ & 0.009 & $1.20[1.08-1.31]$ & $<0.001$ \\
\hline Diabetes & $1.07[0.93-1.21]$ & 0.34 & - & - \\
\hline Chronic disease & $1.08[0.97-1.19]$ & 0.17 & $1.09[0.98-1.20]$ & 0.12 \\
\hline \multicolumn{5}{|l|}{ Type of hysterectomy } \\
\hline Laparoscopy & 1 & - & 1 & - \\
\hline Laparotomy & $0.89[0.75-1.04]$ & 0.12 & $0.86[0.71-0.99]$ & 0.06 \\
\hline Lymphadenectomy & $1.04[0.93-1.15]$ & 0.45 & - & - \\
\hline Adjuvant treatment & $1.07[0.96-1.19]$ & 0.24 & - & - \\
\hline Before 2011 & $1.09[0.98-1.20]$ & 0.13 & $1.11[1.01-1.22]$ & 0.04 \\
\hline \multicolumn{5}{|l|}{ Institutional factors } \\
\hline \multicolumn{5}{|l|}{ Type of institution } \\
\hline Public non-university & 1 & - & 1 & - \\
\hline University & $1.16[0.95-1.37]$ & 0.17 & $1.19[0.98-1.41]$ & 0.10 \\
\hline Private & $1.17[1.04-1.29]$ & 0.02 & $1.20[1.08-1.32]$ & 0.01 \\
\hline \multicolumn{5}{|c|}{ Endometrial cancer activity during 10 years } \\
\hline$<200$ & 1 & - & 1 & - \\
\hline$>=200$ & $0.82[0.70-0.94]$ & 0.001 & $0.81[0.69-0.92]$ & 0.003 \\
\hline
\end{tabular}

CI: Confidence interval.

follow-up $(\mathrm{OR}=1.20[95 \% \mathrm{CI}=1.08-1.31], p<0.01)$. At the individual level, there was better adherence to follow-up guidelines in women who underwent surgery after 2011 $(\mathrm{OR}=1.11[95 \% \mathrm{CI}=1.01-1.22], p=0.04)$. At the institutional level, patients who underwent surgery in private care institutions had an increased risk of a gap between recommendations and practice $(\mathrm{OR}=1.20 \quad[95 \% \mathrm{CI}=1.08$ 1.32], $p=0.01)$ compared to those who underwent surgery in non-university public institutions. There was no difference between the non-university and university public institutions. Treatment in an institution that performed 20 or more hysterectomies for EC per year was significantly associated with a lower incidence of a gap between recommendations and practice $(\mathrm{OR}=0.81$ [95\% $\mathrm{CI}=0.69-0.92], p=0.003)$.

\section{Discussion}

This study shows that the gap between recommendations and practices during follow-up after hysterectomy for EC is present in almost half the patients in France. This risk is increased in patients with EC LDD declaration, patients who underwent surgery before 2011 , in a private health care institution or in an institution that performed less than 20 hysterectomies for EC per year. Patients with factors presumably associated with more aggressive EC (adjuvant therapy and/or surgery by laparotomy and/or having lymph node dissection) did not experience a gap between recommendations and practices more often. EC is a cancer with good prognosis and a recurrence rate of approximately 10\%. ESMO and French recommendations regarding follow-up remain unchanged since 2000 and recommend no further complementary examination in case of a normal physical examination (14). Follow-up after treatment is important for the diagnosis of recurrences that occur mainly in the first 3 years (between 68 and $100 \%$ of cases) and are mostly symptomatic $(70 \% \quad[95 \% \mathrm{CI}=65-75]) \quad(14)$. The recommendation not to make systematically complementary investigations during EC follow-up is based on various studies. In a systematic review published in 2006 , the asymptomatic recurrences detection rate is very inconsistent for these complementary exams, and clinical examination appears to be the most efficient method for the detection of asymptomatic recurrence $(5-33 \%)$ (8). Vaginal cytology and chest X-rays do not appear to be effective in detecting asymptomatic recurrences (vaginal cytology: 0-4\%, chest X-rays: 0-14\%). Abdominal ultrasonography and abdominal pelvic CT scan appear to be relatively modest for the detection of asymptomatic recurrences. CT scans also appear to not be very effective in detecting symptomatic recurrences. Connor et al. reported that $30 \%$ of women with a clinical suspicion of, who finally had a recurrence were not diagnosed with computed tomography (15). The CA 125 assay appears to have diagnostic value only in case of a negative result, but the available data are limited (16). For 
these reasons, physical examination was selected as the only systematic follow-up method for EC with a frequency of 2 to 3 times per year in the first three years after the diagnosis. In a recent Japanese study, Yoshiba et al. found that there were no differences in the overall survival and the overall survival after recurrence between the women with symptoms and without symptoms at recurrence (17). In Japan, the EC follow-up commonly includes physical examination, vaginal cytology, transvaginal ultrasonography, the measurement of tumor markers, and computed tomography. This finding suggests that this intensive routine follow-up for asymptomatic women after treatment for EC does not improve the prognosis of patients with recurrence, as previously indicated by a few studies in North America $(6,18)$.

Thus far, few studies have investigated the adherence to follow-up recommendations in clinical practice in oncology. Brun et al. showed that for the surgical management of stage 1 EC, adherence to the guidelines was low (19). Regarding followup, a Dutch study in 2013 assessed care consumption during follow-up after early stage EC and showed an overconsumption of follow-up care between $19 \%$ and $27 \%$ depending on the year. This consumption was linked to comorbidities, to a higher anxiety score and to participating hospitals, reflecting a difference in the dissemination of guidelines (12).

In the present study, the gap between recommendations and practices increased for patients declared in LDD for EC. These patients represented $66 \%$ of our cases and with $100 \%$ supported care cost were possibly more likely to consume more and to require additional examinations. Among the other $34 \%, 40 \%$ had an LDD for another disease. The updated recommendations for EC were published in November 2010 (14). The recommendations for follow-up remained the same compared to 2000: to practice only systematic physical examination. However, the finding that patients who underwent surgery before 2011 experienced more differences in practices suggests that this update had an impact on the adherence to recommendations.

However, several limitations must be discussed. The EGB database does not permit access to clinical and histological data. Thus, we did not have enough information on the disease stage and grade, and therefore the risk of recurrence (20). No information was available on the reasons for achieving investigations. Contrary to vaginal smear, which is never recommended in EC follow-up, imaging and CA 125 evaluations can be prescribed in another context than for the follow-up of EC. For example, these tests can be performed in patients with associated cancer, or in the follow-up of a case suspicious of EC recurrence. For these reasons, we decided to exclude patients with associated cancer or with a probable recurrence. Moreover, patients who had adjuvant treatment and therefore a more advanced cancer had no more additional tests during the follow-up. Another limitation could be the small number of patients included in our study. The database is a sample of about 1 percent of the population, and the absence of clinical data required exclusion criteria, which reduced the initial number. Nevertheless, this database is a representative sample of the French population protected by health insurance.

Despite these limitations, our database was representative and appropriate for the study of the follow-up for EC in France. The incidence of EC has been stable for 30 years in France (21). Due to the steady growth of the French population, the incidence of EC in France has increased progressively (6171 new cases in 2005 to 7275 new cases in 2012). The number of cases theoretically expected from the EGB database between 2005 and 2014 based on the incidence of EC was approximately 65 cases per year or 650 cases over 10 years. We identified 547 cases from the EGB database. This small difference can be due to the fact that the identification of cases was based on recording of common classification medical procedures that can sometimes be mis-coded. Regarding the sociodemographic characteristics of cases, the results are consistent with the characteristics of women with EC in France for the age and the existence of associated diabetes. The distribution and the mortality rates were consistent with the literature data. The 5year overall survival rates ranged from $74 \%$ to $91 \%$ (22); we have demonstrated a survival rate at 5 years of $78 \%$ and a 2 year survival rate of $87 \%$.

In conclusion, the present study suggests that there is a gap between recommendations and practices during the follow-up of EC in almost half of the cases in France. The risk increases in patients reported in LDD for EC, in patients who underwent surgery before the recommendation update at the end of 2010 , and in patients supported in a private health institution that have performed fewer than 200 ECs over 10 years. Thus, improved diffusion and communication of recommendations in health institutions that care for patients with EC may improve these rates.

\section{References}

1 Hüsing A, Dossus L, Ferrari P, Tjønneland A, Hansen L, Fagherazzi G, Baglietto L, Schock H, Chang-Claude J, Boeing H, Steffen A, Trichopoulou A, Bamia C, Katsoulis M, Krogh V, Palli D, Panico S, Onland-Moret NC, Peeters PH, Bueno-deMesquita HB, Weiderpass E, Gram IT, Ardanaz E, ObónSantacana M, Navarro C, Sánchez-Cantalejo E, Etxezarreta N, Allen NE, Khaw KT, Wareham N, Rinaldi S, Romieu I, Merritt MA, Gunter M, Riboli E and Kaaks R: An epidemiological model for prediction of endometrial cancer risk in Europe. Eur J Epidemiol 31: 51-60, 2016.

2 Colombo N, Preti E, Landoni F, Carinelli S, Colombo A, Marini $\mathrm{C}$ and Sessa C; ESMO Guidelines Working Group: Endometrial cancer: ESMO Clinical Practice Guidelines for diagnosis, treatment and follow-up. Ann Oncol 24: vi33-38, 2013.

3 Salvesen HB, Akslen LA, Iversen T and Iversen OE: Recurrence of endometrial carcinoma and the value of routine follow up. $\mathrm{Br}$ J Obstet Gynaecol 104: 1302-1307, 1997. 
4 Amant F, Mirza MR, Koskas M and Creutzberg CL: Cancer of the corpus uteri. Int J Gynaecol Obstet Off Organ Int Fed Gynaecol Obstet 131: S96-104, 2015.

5 Brémond A, Bataillard A, Thomas L, Achard J-L, Fervers , Fondrinier E, Lansac J, Bailly C, Hoffstetter S, d'Anjou J, Descamps P, Farsi F, Jean-Paul G, Laffargue F, Rodier JF, Vincent $\mathrm{P}$ and Pigneux J: Standards, Options and Recommendations 2000: non metastatic endometrial cancer]. Bull Cancer (Paris) 89(7-8): 697-706, 2002.

6 Shumsky AG, Stuart GC, Brasher PM, Nation JG, Robertson DI and Sangkarat S: An evaluation of routine follow-up of patients treated for endometrial carcinoma. Gynecol Oncol 55(2): 229-233, 1994.

7 Ackerman I, Malone S, Thomas G, Franssen E, Balogh J and Dembo A: Endometrial carcinoma - relative effectiveness of adjuvant irradiation vs. therapy reserved for relapse. Gynecol Oncol 60(2): 177-183, 1996.

8 Fung-Kee-Fung M, Dodge J, Elit L, Lukka H, Chambers A and Oliver T: Follow-up after primary therapy for endometrial cancer: a systematic review. Gynecol Oncol 101(3): 520-529, 2006.

9 Allsop JR, Preston J and Crocker S: Is there any value in the long-term follow up of women treated for endometrial cancer? Br J Obstet Gynaecol 104(1): 122, 1997.

10 Kew FM, Roberts AP and Cruickshank DJ: The role of routine follow-up after gynecological malignancy. Int J Gynecol Cancer Off J Int Gynecol Cancer Soc 15(3): 413-419, 2005.

11 Salani R, Nagel CI, Drennen E and Bristow RE: Recurrence patterns and surveillance for patients with early stage endometrial cancer. Gynecol Oncol 123(2): 205-207, 2011.

12 Nicolaije KA1, Ezendam NP, Vos MC, Boll D, Pijnenborg JM, Kruitwagen RF, Lybeert ML and van de Poll-Franse LV: Followup practice in endometrial cancer and the association with patient and hospital characteristics: a study from the populationbased PROFILES registry. Gynecol Oncol 129(2): 324-331, 2013.

13 De Roquefeuille L, Studer A, Neumann A and Merlière Y: The Echantillon généraliste des bénéficiaires: representativeness, scope and limits. Prat Organ Soins 40(3): 213-223, 2009.

14 French National Cancer Institute: INCa, professional recommendations. Endometrial Cancer, 2010.
15 Connor JP, Andrews JI, Anderson B and Buller RE: Computed tomography in endometrial carcinoma. Obstet Gynecol 95(5): 692-696, 2000.

16 Saga T, Higashi T, Ishimori T, Mamede M, Nakamoto Y, Mukai T, Fujita T, Togashi K, Yura S, Higuchi T, Kita M, Fujii S and Konishi J:Clinical value of FDG-PET in the follow up of postoperative patients with endometrial cancer. Ann Nucl Med 17(3): 197-203, 2003.

17 Yoshiba T, Takei Y, Machida S, Taneichi A, Sato N, Takahashi S, Takahashi Y, Saga Y, Matsubara S and Fujiwara H: Prognosis of endometrial cancer patients with and without symptoms at recurrence. J Obstet Gynaecol Res 42(12): 1814-1821, 2016.

18 Agboola OO, Grunfeld E, Coyle D and Perry GA: Costs and benefits of routine follow-up after curative treatment for endometrial cancer. CMAJ Can Med Assoc J J Assoc Medicale Can 157(7): 879-878, 1997.

19 Brun J-L, Ouldamer L, Bourdel N, Huchon C, Koskas M and Gauthier T: Management of stage I endometrial cancer in France: a survey on current practice. Ann Surg Oncol 22(7): 2395-2400, 2015.

20 Koskas M, Chabbert-Buffet N, Bendifallah S, Luton D, ClavelChapelon F and Rouzier R: Prognostic value of the 2009 FIGO staging for endometrial cancer: an illustration of the E3N cohort. Int J Gynecol Cancer Off J Int Gynecol Cancer Soc 22(3): 447451, 2012.

21 Binder-Foucard F, Belot A, Delafosse P, Remontet L, Woronoff A and Bossard N: National estimate of cancer incidence and mortality in France between 1980 and 2012 Study based on cancer registries in the Francim network - Part 1: solid tumors. Rev Epidemiol Sante Publique 62(2): 95-108, 2014.

22 Morice P, Leary A, Creutzberg C, Abu-Rustum N and Darai E: Endometrial cancer. Lancet Lond Engl 387(10023): 1094-1108, 2016. 